\title{
shinyJackpot: Visualizing lottery gambling in a large Canadian city
}

\author{
Andrew $\mathrm{Li}^{1,2}$ \\ 1 Department of Psychology, University of British Columbia \\ 2 Djavad Mowafaghian Centre for Brain Health, University of British Columbia \\ andrewc.li@ubc.ca
}

\begin{abstract}
Lottery gambling is widely enjoyed by Canadians and is the most popular form of legal gambling. As such, discovering and analyzing patterns in lottery gambling data is an important but nontrivial task. In this work, we describe the theory and development of this shiny web application. Three methods were presented to process and visualize it to the end user to allow for faster pattern discovery. Firstly, a bubble plot was utilized for the comparative analysis of lottery sales per each neighbourhood of the city of Toronto, Canada. Secondly, a scatter plot was used to explore the relationship between different neighbourhoods, lottery game product, year, lottery ticket sales, and demographic information. Lastly, a line graph was deployed to compare the jackpot size and ticket sales over time. shinyJackpot is deployed at https: //andrewcli.shinyapps.io/shinyJackpot/for online use.
\end{abstract}

Keywords: Gambling · Lottery · R Shiny · ggplot2 · Visualization.

\section{Introduction}

Gambling is a widespread leisure activity that causes significant harm and cost. For some individuals, it has a direct financial impact that affects family members and friends. Lottery gambling has become so popular and enticing because of its low cost and potential prize reward [2]. However, lower income individuals spend a greater proportion of their income on lottery gambling than those with higher income [3, 7, 15, 16. Furthermore, ticket sales not only correlated with levels of income, but also with lower socioeconomic status (SES) 10,13. As such, it is important to visualize and explore this data for analysis by experts or regular users who wish to discover trends [6]. Graphics enable the finding of patterns and relationships to reveal insights into dense and complex data [4].

Creating web applications is not trivial as it requires proficiency in several programming languages. However, the introduction of the R package Shiny [5] offers new possibilities for web applications because it only requires familiarity with the $\mathrm{R}$ programming language to create an interactive web app. Furthermore, the transition from static to dynamic graphics is relatively straightforward for those who have knowledge of $\mathrm{R}[22$. The use of Shiny has seen a large growth (see Fig. 11. For example, Indiana State 8], Rice [12], and Berkeley [19] University has 
a wide collection of apps to augment traditional statistic courses. As well, Shiny apps are well used in the field of bioinformatics for dynamic data visualization. Some examples include shinyCircos to visualize genomic data with circos plots 23 and shinyChromosome to produce non-circular whole genome diagrams [24]. However, the use of Shiny apps in psychology have been limited 1, 9, 18]. To the best of our knowledge, only one visualization exists for lottery data. Local Lotto is offered by the Civic Data Design Lab at MIT 14. The tool provides geographic distribution of percentage of income spend on lottery tickets. However, it is limited to Brooklyn, New York and is unavailable at this time. As such we propose a new tool, shinyJackpot, a shiny app that provides lottery gambling data at the city level and provides interactive data visualization tools that allows users to explore trends in the cost attributable to lottery gambling in Toronto, Canada.

We hope that this tool would be of interest to data analysts exploring related questions, policy makers interested in past trends or the general public who wonder about the lottery gambling rate in their neighbourhood and how it compares to other parts of the city.

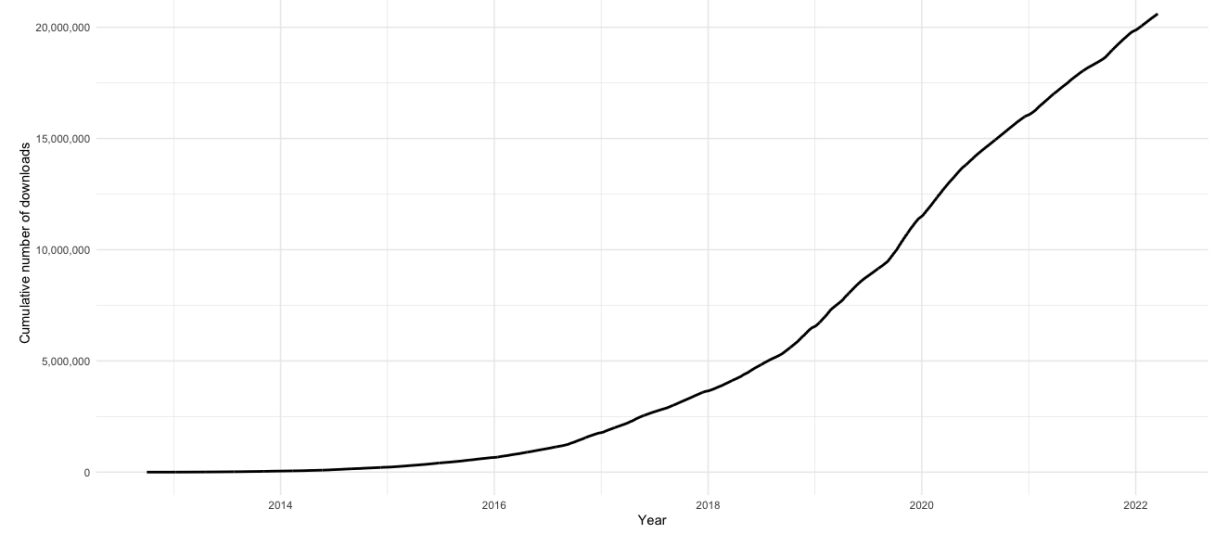

Fig. 1. The cumulative number of Shiny package downloads from 2012-10-01 to 202203-17. Data was generated by RStudio as they publish the download logs from their CRAN package mirror daily at http://cran-logs.rstudio.com/.

\section{Implementation}

shinyJackpot was built almost entirely with the $\mathrm{R}$ programming language 20 and is extended using HTML and CSS. shinyJackpot was primarily intended to be used on its shiny server via its URL as users only need to need to have a web browser installed. However, it can be hosted on and launched locally on 
any system with $\mathrm{R}$ installed provided the prerequisite packages are installed. the latest version can be cloned or forked via the GitHub page. The latest version can be launched as follows:

shiny: $:$ runGitHub (repo = "andr3wli/shinyapps",

subdir = "shinyJackpot")

\section{Features}

\section{$3.1 \quad$ Intro}

The Intro tab provides insights and motivations as to why we created this shiny app. Furthermore, it includes the contact information of the author and maintainer as well as acknowledgements.

\subsection{Map}

As shown in Fig. 2, the Map tab visualizes the number of tickets purchased by neighbourhood. It uses a bubble plot over a map of Toronto with the size of the bubble indicating the numbers of ticket purchased. The different colors represent different boroughs roughly corresponding to: Central Toronto, Downtown Toronto, East York, Etobicoke, North York, Scarborough, West Toronto, and York. The map was generated via the leaflet package [11. The radii of the circle represent the number of tickets sold in the neighbourhood - larger circles represents more tickets sold. Users can navigate the map with their mouse and interact with the bubbles to see the exact number of tickets purchased for a specific neighbourhood.

The use of the bubble plot over the map allowed for rapid and easy interpretation of the geographic areas with the greatest number of lottery ticket purchases.

\subsection{Relationship}

As seen in Fig. 3, the Relationship tab allows users to visualize and explore the relationships between the different variables. Using a side bar panel, users can select the specific lottery game(s), year(s), and borough(s) to visualize. Next, users select either ticket sales or net sales for the outcome variable. Then, users can select income, education, socioeconomic status, population, or MBSA as the demographic variable. Lastly, users can choose to add a best fit line of the city, multiple lines for the boroughs, or both. A plot and the correlation is reported from the selected variables. The plot is produced via the ggplot2 package 21] and is made interactive via the ggiraph package. 


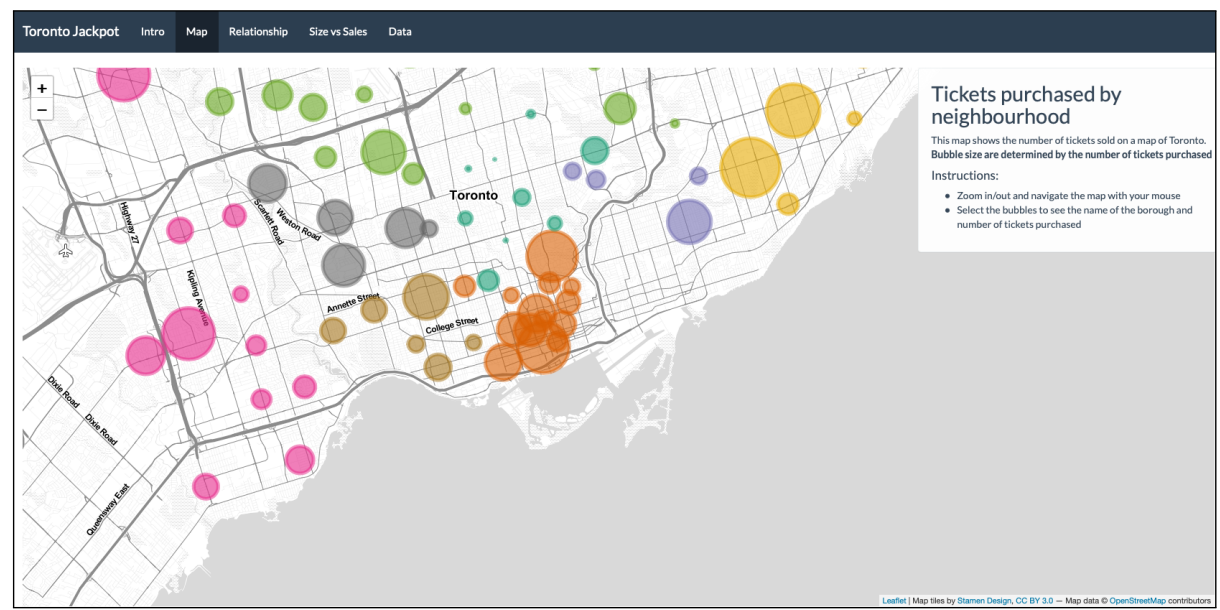

Fig. 2. User interface of the Map tab. The bubbles are interactive and allow users to gain more information when they hover over a specific bubble.

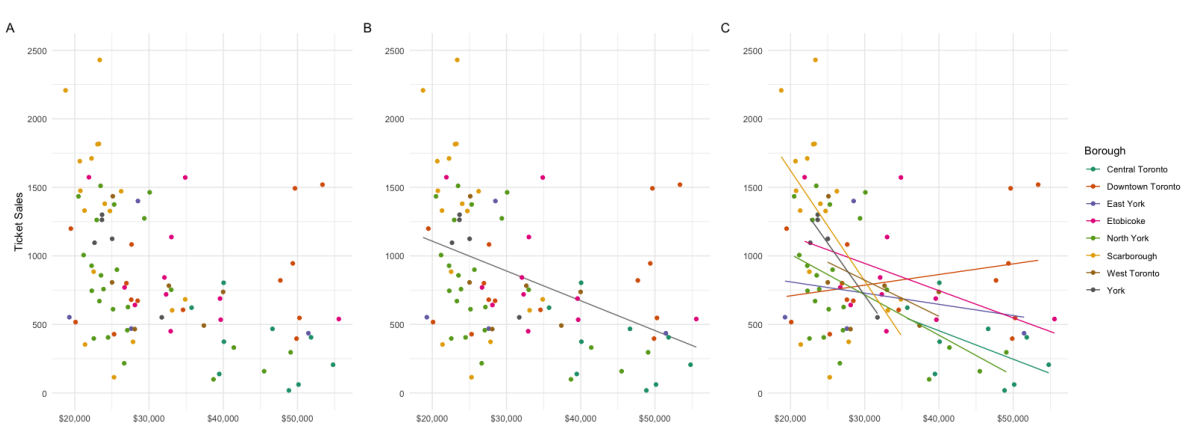

Fig. 3. The Relationship tab allows users to explore the relationship between different demographic variables and ticket sales or net sales. This example has income on the $\mathrm{x}$-axis and ticket sales on the $\mathrm{y}$-axis. Furthermore, users can select no line (A), a single best fit line (B), or multiple best fit lines (C).

\subsection{Size vs. Sales}

The Size vs. Sales (Fig. (4) tab visualizes the relationship between the jackpot size and the ticket sales. Users can select the specific year and the specific lottery game to explore. As well, users can track this relationship yearly, monthly, or weekly. A plot of the jackpot size and the ticket sales are presented, and users can clearly and easily see the change in ticket sales and jackpot size. Again, these plots are generated with the ggplot2 package 21. 


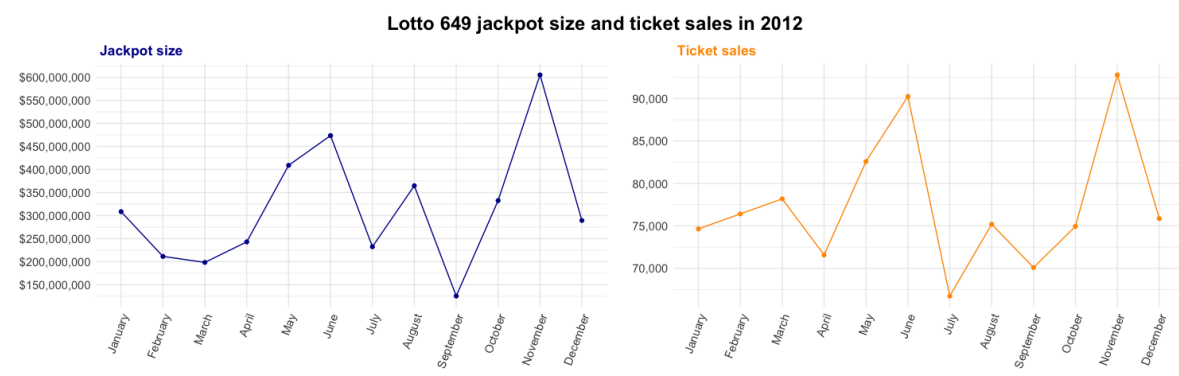

Fig. 4. The Size vs Sales tab allows users to explore and visualize the relationship between jackpot size and jackpot sales. This example shows the monthly size and sales from Lotto 649 in 2012.

\subsection{Data}

The data tab offers users the opportunity to explore the data set interactively via the side panel. Here, users can filter through the year, lottery game, and borough(s). The filtered data set will be made available to explore on the app or for download for further analysis.

\section{Evaluation}

shinyJackpot is still under active development, as such, the evaluation of its capabilities is still in the early stages. However, the goals and the target users of shinyJackpot is well-defined: to enable researchers to explore related questions related to gambling and to enable users to explore lottery gambling rate in Toronto. To this end, we can evaluate the usability of this web application. Our approach to usability included multiple rounds of testing to improve weaknesses and to find potential bugs [17. Qualitative data was collected over multiple months with four psychology researchers. This was invaluable as the testing revealed several errors and contributed to the current look and functionality of the app. The feedback provided by testers greatly improved the use and overall quality of shinyJackpot.

\section{$5 \quad$ Licensing and Availability}

shinyJackpot is licenced under the GNU General Public Licence (v3.0). All its source code was made publicly available in its GitHub repository (https://github. com/andr3wli/shinyapps). Furthermore, there is a corresponding issue tracker for bug reporting and feature enhancements. Requests for fixes, feature updates, general questions and concerns can be made by filling an issue (https://github. com/andr3wli/shinyapps/issues). Lastly, users are encouraged to contribute to the development of shinyJackpot. 


\section{Acknowledgement.}

Geocoding data used in the Map tab was made available by https://geocoder.ca.

\section{References}

1. Andrews, S., Ellis, D.A., Shaw, H., Piwek, L.: Beyond self-report: Tools to compare estimated and real-world smartphone use. PloS one 10(10), e0139004 (2015)

2. Ariyabuddhiphongs, V.: Lottery gambling: A review. Journal of Gambling Studies 27(1), 15-33 (2011)

3. Beckert, J., Lutter, M.: The inequality of fair play: lottery gambling and social stratification in germany. European sociological review 25(4), 475-488 (2009)

4. Chambers, J.M., Cleveland, W.S., Kleiner, B., Tukey, P.A.: Graphical methods for data analysis. Chapman and Hall/CRC (2018)

5. Chang, W., Cheng, J., Allaire, J., Sievert, C., Schloerke, B., Xie, Y., Allen, J., McPherson, J., Dipert, A., Borges, B.: shiny: Web Application Framework for R (2021), https://CRAN.R-project.org/package=shiny, r package version 1.6.0

6. Chen, C.h., Härdle, W.K., Unwin, A.: Handbook of data visualization. Springer Science \& Business Media (2007)

7. Clotfelter, C.T., Cook, P.J.: Implicit taxation in lottery finance. National Tax Journal 40(4), 533-546 (1987)

8. DePaolo, C.A.: The stat-attic website: Links to statistics applets for introductory courses. Journal of Statistics Education 18(3) (2010)

9. Ellis, D.A., Renouf, K.J.: Predicting fear of crime: Personality outperforms prior victimisation. The Journal of Forensic Psychiatry \& Psychology 29(3), 403-418 (2018)

10. Fu, H.N., Monson, E., Otto, A.R.: Relationships between socio-economic status and lottery gambling across lottery types: neighborhood-level evidence from a large city. Addiction 116(5), 1256-1261 (2021)

11. Graul, C.: leafletR: Interactive Web-Maps Based on the Leaflet JavaScript Library (2016), http://cran.r-project.org/package=leafletR, r package version 0.4-0

12. Lane, D.M.: The rice virtual lab in statistics. Behavior Research Methods, Instruments, \& Computers 31(1), 24-33 (1999)

13. Lang, K.B., Omori, M.: Can demographic variables predict lottery and pari-mutuel losses? an empirical investigation. Journal of Gambling Studies 25(2), 171-183 (2009)

14. Lim, V., Deahl, E., Rubel, L., Williams, S.: Local lotto (2015)

15. Livernois, J.R.: The redistributive effects of lotteries: Evidence from canada. Public Finance Quarterly 15(3), 339-351 (1987)

16. Miyazaki, A.D., Hansen, A., Sprott, D.E.: A longitudinal analysis of income-based tax regressivity of state-sponsored lotteries. Journal of Public Policy \& Marketing $\mathbf{1 7}(2), 161-172(1998)$

17. Rubin, J., Dana, J.S.D.C.C.: Chisnell: Handbook of usability testing (2008)

18. Shaw, H., Ellis, D.A., Kendrick, L.R., Ziegler, F., Wiseman, R.: Predicting smartphone operating system from personality and individual differences. Cyberpsychology, Behavior, and Social Networking 19(12), 727-732 (2016)

19. Stark, P.B.: Sticigui: statistics tools for internet and classroom instruction with a graphic user interface. Available at Web site: http://stat-www. berkeley. edu/users/stark/SticiGui/index. htm (8 August 2004) (2004) 
20. Team, R.C., et al.: R: A language and environment for statistical computing (2013)

21. Wickham, H.: ggplot2: Elegant Graphics for Data Analysis. Springer-Verlag New York (2016), https://ggplot2.tidyverse.org

22. Xie, Y.: animation: An $\mathrm{r}$ package for creating animations and demonstrating statistical methods. Journal of Statistical Software 53, 1-27 (2013)

23. Yu, Y., Ouyang, Y., Yao, W.: shinycircos: an $\mathrm{r} /$ shiny application for interactive creation of circos plot. Bioinformatics 34(7), 1229-1231 (2018)

24. Yu, Y., Yao, W., Wang, Y., Huang, F.: shinychromosome: an r/shiny application for interactive creation of non-circular plots of whole genomes. Genomics, Proteomics \& Bioinformatics 17(5), 535-539 (2019) 